\title{
Off with a Bang! Introducing the Journal of Natural Health Product Research
}

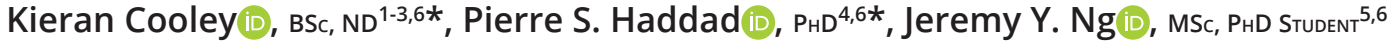 \\ ${ }^{1}$ Canadian College of Naturopathic Medicine, Toronto, Ontario, Canada \\ ${ }^{2}$ Australian Research Center in Complementary and Integrative Medicine, University of Technology, Ultimo, Australia \\ ${ }^{3}$ Pacific College of Oriental Medicine, San Diego, California, United States of America \\ ${ }^{4}$ Department of Pharmacology and Physiology, Université de Montréal, Montreal, Quebec, Canada \\ ${ }^{5}$ Department of Health Research Methods, Evidence, and Impact, Faculty of Health Sciences, McMaster University, \\ Hamilton, Ontario, Canada \\ ${ }^{6}$ NHP Publications, Toronto, Ontario, Canada \\ *®editor@jnhpresearch.com
}

KEYWORDS: natural health product; NHP; natural product; herbal; JNHPR; Health Canada; Natural and

Non-prescription Health Products Directorate; complementary and alternative medicine; traditional medicine;

dietary supplements

\section{Introducing the Journal of Natural Health Product Research}

This editorial serves to introduce the Journal of Natural Health Product Research (JNHPR), published by NHP Publications based out of Toronto, Canada; the JNHPR is the world's first journal focusing exclusively on the publication of high-quality, peer-reviewed, open access and multidisciplinary research on natural health products (NHPs). Furthermore, NHP Publications is supported in part by the Federal Government of Canada's Department of Canadian Heritage, which fittingly aligns with the fact that Canada is a world leader in NHP research. While the term "natural health product" is primarily a Canadian one, we certainly welcome submission to JNHPR of manuscripts from around the globe, including those with international or cross-regional perspectives. Locally, the definition of NHP used by Health Canada captures six categories as follows: vitamins and minerals; herbal remedies; homeopathic medicines; traditional medicines like traditional Chinese and Ayurvedic (East Indian) medicines; probiotics; and other products like amino acids, essential fatty acids and other components isolated or purified from natural sources [1]. We feel that this definition encompasses the general scope for our journal as being inclusive of the broad nature of these substances including the spectrum of agricultural practices, biochemical properties, physiological effects, manufacturing, use as functional foods or medicines, or use, knowledge and beliefs amongst users and non-users alike. Many of these substances also fall within the realm of "dietary supplements", "traditional", "complementary", "functional" or "alternative" medicines [1]. The Canadian context is particular in that all NHPs must adhere to regulations set forth by the Natural and Non-prescription Health Products Directorate (NNHPD) before entering the market. Indeed, the NNHPD is the authority responsible for regulating the sale of NHPs in Canada. Notably, it was tasked by the Canadian government with the role of ensuring that "Canadians have ready access to natural health products that are safe, effective and of high quality while respecting freedom of choice and philosophical and cultural diversity" [2]. It is also, to our knowledge, the only regulatory agency worldwide to be mandated to foster research on the products it regulates. When it was instated in 2000, the NNHPD (previously the Natural Health Products Directorate) brought together many Canadian scientists doing research on NHPs to advise them on that matter. It induced a momentum that lead to the creation of the Natural Health Product Research Society (NHPRS) of Canada in 2003. The JNHPR notably shares the NHPRS's mission to facilitate and support meaningful, scientifically rigorous research and education in the field of natural health products. 
The idea of regulating NHPs or equivalent products, however, is certainly not unique to Canada. Indeed, many developed and developing countries alike currently seek to address or refine how best to regulate natural substances and products, often derived from traditional medicines existing for hundreds if not thousands of years. In fact, the World Health Organization (WHO) reports that, even today, nearly three-quarters of the world's population rely on traditional medicine, notably associated NHPs, for their primary health care needs [3]. Indeed, NHPs are found in every country in the world, their importance is increasingly appreciated and their demand constantly rising. Many countries are renewing or recognizing the need to facilitate approaches that allow governments, healthcare practitioners and patients access to NHPs in a safe, respectful, cost-efficient and effective manner [3]. This is highlighted by the existence of the WHO's Traditional Medicine Strategy that "aims to support Member States in developing proactive policies and implementing action plans that will strengthen the role traditional medicine plays in keeping populations healthy" [3]. This strategy provides selected examples of notable initiatives by region/country, many of which target products under the NHP definition. Such initiatives range from smaller-scale events or research studies to larger-scale multi-country research networks, or, in the case of countries like Canada, Australia and New-Zealand, to the establishment of comprehensive regulatory frameworks for NHPs and equivalent products as well as harmonized regulatory initiatives [3].

A crucial element of such worldwide initiatives rests on enhancing high-quality scientific research on NHPs. In fact, research relating to the therapeutic properties of numerous NHPs has increased at a staggering rate over recent years [4]. Yet, a rigorous, peer-reviewed, open access journal dedicated to this area is surprisingly lacking. We believe that there is a need to provide NHP researchers with a common publishing and discussion space as they work across multiple disciplines and sectors. NHP research taking place mainly across academic institutions are currently published in a plethora of journals that focus either too strictly on natural products or too broadly on traditional, complementary, alternative and integrative approaches and modalities. Many of these are also not easily accessible to many potential readers, notably in less developed countries with limited scientific library resources. The JNHPR seeks to fill this void by providing NHP researchers with an open-access, comprehensive and high-quality venue for publication and discussion.

In particular, the JNHPR seeks to offer space for equally high-quality NHP research occurring in the private sector. Indeed, the NHP industry in Canada and abroad are actively involved in evidence-based studies detailing product safety and effectiveness, as well as advances in manufacturing, quality control, or post-market surveillance. Yet, scientific journals rarely allocate significant space for industry-driven research. Likewise, government agencies also contribute significant assessments of elements related to NHP research, consumer use or regulation. Again, such valuable information often remains dormant or buried in highly specialized publications. The JNHPR thus aims to become a central publication that encompasses all aspects of NHP research, allowing researchers and other NHP stakeholders to connect and interact in an optimal manner around the discovery, development, manufacturing, marketing, and regulation of NHPs that treat a multitude of diseases and conditions.

Hence, NHP Publications will publish a comprehensive breadth of original research, review articles, research protocols, and reports that relate to the use, quality, safety (including adverse reactions and herbdrug interactions), efficacy, manufacturing (including industrial innovations and emerging technologies) as well as regulation of NHPs regardless of discipline or sector. The JNHPR will also offer a venue for the publication of abstracts and proceedings from NHPrelated research meetings, such as the NHPRS Annual Conference. Also less dealt with in other high-quality journals, the JNHPR will open its pages to null studies and to research exploring barriers and challenges encountered in NHP research. We believe that sharing this type of knowledge, demonstrates rigour and transparency, facilitates a sense of community and, most importantly, catalyzes growth in the field. On another note, the vast majority of conventional healthcare providers generally receive weak or inexistent training and education on NHPs in current academic curricula of the Western hemisphere [5]. The JNHPR thus wishes to promote submissions relating to NHPs in the context of healthcare education. Finally, our journal will foster the publication of case reports on NHPs within the same multidisciplinary framework discussed above. This will bridge another gap and help to build a solid clinical literature on NHPs for healthcare practitioners worldwide to refer to.

Our goal is that this journal will become a primary resource on NHP research for academic, industry and government researchers alike, as well as healthcare practitioners. We will achieve this by providing a central all-inclusive space for authors and readers alike who have a vested interest in advancing knowledge surrounding NHPs. We are excited to launch the JNHPR 
and we greatly look forward to publishing your highest quality contributions to NHP research.

Kieran Cooley, BSc, ND

Co-Editor-in-Chief

Pierre S. Haddad, PhD

Co-Editor-in-Chief

Jeremy Y. Ng, MSc, PhD Student

Journal Manager and Publisher

\section{List of Abbreviations Used}

JNHPR: Journal of Natural Health Product Research

NHP: Natural health product

NHPRS: Natural Health Product Research Society

NNHPD: Natural and Non-prescription Health Products

Directorate

WHO: World Health Organization

\section{Conflicts of Interest}

The author(s) declare that they have no conflicts of interest.

\section{Authors' Contributions}

KC: revised the manuscript critically, and gave final approval of the version to be published

(1D) https://orcid.org/0000-0001-7960-6504

PSH: revised the manuscript critically, and gave final approval of the version to be published

(D) https://orcid.org/0000-0002-8782-5165
JYN: drafted the manuscript, revised the manuscript critically, and gave final approval of the version to be published

(D) https://orcid.org/0000-0003-0031-5873

\section{References}

[1] Government of Canada [Internet]. About Natural Health Products. [cited 2018 Dec 15]. Available from: http:// www.hc-sc.gc.ca/dhp-mps/prodnatur/about-apropos/ cons-eng.php

[2] Government of Canada [Internet]. Natural and Nonprescription Health Products Directorate. [cited 2018 Dec 15]. Available from: http://www.hc-sc.gc.ca/ahc-asc/ branch-dirgen/hpfb-dgpsa/nhpd-dpsn/index-eng.php

[3] World Health Organization [Internet]. WHO Traditional Medicine Strategy 2014-2023. [cited 2018 Dec 15]. Available from: http://apps.who.int/iris/bitstr eam/10665/92455/1/9789241506090_eng.pdf

[4] Government of Canada [Internet]. Results from the Functional Foods and Natural Health Products Survey (2011). [cited 2018 Dec 15]. Available from: http://www.agr.gc.ca/eng/industry-markets-andtrade/canadian-agri-food-sector-intelligence/ functional-foods-and-natural-health-products/ trends-and-market-opportunities-for-the-functionalfoods-and-natural-health-products-sector/resultsfrom-the-functional-foods-and-natural-health-products-survey-2011/?id=1387481727299

[5] Frass M, Strassl RP, Friehs H, Müllner M, Kundi M, Kaye AD. Use and acceptance of complementary and alternative medicine among the general population and medical personnel: a systematic review. The Ochsner Journal. 2012;12(1):45-56. https://www.ncbi.nlm.nih.gov/pmc/ articles/PMC3307506/

\section{Article Information}

Managing Editor: Kieran Cooley, Pierre Haddad

Peer Reviewers: This article was not peer reviewed.

Article Dates: Published Jan 3019

\section{Citation}

Please cite this article as follows:

Cooley K, Haddad PS, Ng JY. Off with a bang! Introducing the Journal of Natural Health Product Research. Journal of Natural Health Product Research. 2019 Jan 30: Vol1 (1). https://jnhpresearch.com/index.php/jnhpr/article/view/4 DOI Link: https://doi.org/10.33211/jnhpr.4

\section{Copyright}

(c) Kieran Cooley, Pierre S. Haddad, Jeremy Y. Ng. (2019). Published first in the Journal of Natural Health Product Research. This is an open access article distributed under the terms of the Creative Commons Attribution License (https://creativecommons.org/licenses/by/4.0/), which permits unrestricted use, distribution, and reproduction in any medium, provided the original work, first published in the Journal of Natural Health Product Research, an NHP 
Publications journal, is properly cited. The complete bibliographic information, a link to the original publication on https://www.jnhpresearch.com, as well as this copyright and license information must be included.

Journal of Natural Health Product Research
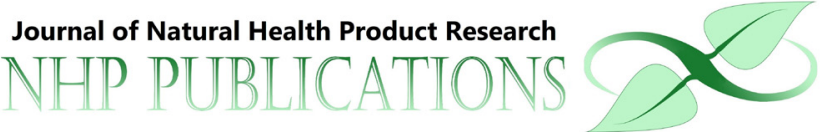

Canadà̀

Does the safety, efficacy, and quality of natural health products matter to YOU?

Submit your research article to the Journal of Natural Health Product Research!

Pre-submission inquiries? Send us an email at editorial.office@jnhpresearch.com

Facebook, Twitter and LinkedIn: @NHPPublications 